\title{
Directly Observing Squeezed Phonon States with Femtosecond X-Ray Diffraction
}

\author{
S. L. Johnson, ${ }^{1, *}$ P. Beaud, ${ }^{1}$ E. Vorobeva, ${ }^{1}$ C. J. Milne, ${ }^{2}$ É. D. Murray, ${ }^{3}$ S. Fahy, ${ }^{4}$ and G. Ingold ${ }^{1}$ \\ ${ }^{1}$ Swiss Light Source, Paul Scherrer Institut, 5232 Villigen PSI, Switzerland \\ ${ }^{2}$ Laboratoire de Spectroscopie Ultrarapide, Ecole Polytechnique Fédérale de Lausanne, 1015 Lausanne, Switzerland \\ ${ }^{3}$ Department of Physics and Astronomy, Rutgers University, Piscataway, New Jersey 08854-8019, USA \\ ${ }^{4}$ Tyndall National Institute and Department of Physics, University College, Cork, Ireland
}

(Received 13 December 2008; published 27 April 2009)

\begin{abstract}
Squeezed states are quantum states of a harmonic oscillator in which the variance of two conjugate variables each oscillate out of phase. Ultrafast optical excitation of crystals can create squeezed phonon states, where the variance of the atomic displacements oscillates due to a sudden change in the interatomic bonding strength. With femtosecond x-ray diffraction we measure squeezing oscillations in bismuth and conclude that they are consistent with a model in which electronic excitation softens all phonon modes by a constant scaling factor.
\end{abstract}

DOI: 10.1103/PhysRevLett.102.175503

PACS numbers: 63.20.K-, 61.80.Ba, 78.47.J-

Squeezed states, where the variance of two conjugate variables oscillate out of phase in time, are a centerpiece of modern quantum optics [1,2]. The physics of squeezing is not limited to the electromagnetic field, however, and indeed there has been much interest in the squeezing of various bosons encountered in condensed matter physics. In particular, the idea of squeezing the vibrational phonon modes of a crystal has attracted attention [3-6]. If, for example, a laser pulse with a duration much shorter than a phonon period excites a crystal rapidly into a state where the phonon frequency is different, squeezed states of the phonon field may result. Experimental investigation of phonon squeezing has focused predominantly on optical preparation and detection, relying on either subtle nonlinear interaction of the phonons with light via a second order Raman susceptibility tensor $[3,6]$ or by careful analysis of the statistics in time-resolved optical reflectivity measurements [5].

An alternative approach for observing phonon squeezing uses femtosecond time-resolved diffraction of $\mathrm{x}$ rays to measure the variance of atomic positions. In kinematic x-ray diffraction theory, the intensity $I$ of a diffraction peak with reciprocal lattice vector $\mathbf{G}$ is

$$
I \propto\left|\sum_{j} f_{j} e^{i \mathbf{G} \cdot\left\langle\hat{\mathbf{r}}_{j}\right\rangle} e^{-(1 / 2)\left\langle\left(\hat{\mathbf{u}}_{j} \cdot \mathbf{G}\right)^{2}\right\rangle}\right|^{2},
$$

where the index $j$ runs over all atoms in a unit cell, $f_{j}$ is the atomic scattering factor of atom $j, \hat{\mathbf{r}}_{j}$ is the position operator of atom $j$ within the unit cell, and $\hat{\mathbf{u}}_{j}$ are the atomic displacement operators [7]. Written in terms of phonon coordinate operators $\hat{Q}_{\mathbf{k} s}$ with wave vector $\mathbf{k}$ and branch index $s$, we have $\left\langle\hat{\mathbf{r}}_{j}\right\rangle=\sum_{s}\left\langle\hat{Q}_{\mathbf{0}_{s}}\right\rangle \boldsymbol{\epsilon}_{\mathbf{0} s}^{j}$ and $\left\langle\left(\hat{\mathbf{u}}_{j} \cdot \mathbf{G}\right)^{2}\right\rangle=$ $\frac{1}{N} \sum_{\mathbf{k} s s^{\prime}}\left\langle\hat{Q}_{\mathbf{k} s} \hat{Q}_{-\mathbf{k} s^{\prime}}\right\rangle\left(\boldsymbol{\epsilon}_{\mathbf{k} s}^{j} \cdot \mathbf{G}\right)\left(\boldsymbol{\epsilon}_{-\mathbf{k} s^{\prime}}^{j} \cdot \mathbf{G}\right)$, where $\boldsymbol{\epsilon}_{\mathbf{k} s}^{j}$ is the phonon eigenvector and $N$ is the total number of unit cells. The first exponential factor in Eq. (1) is then sensitive to $\left\langle\hat{Q}_{0 s}\right\rangle$ and thus to $\mathbf{k}=0$ coherent optical phonons. The second exponential factor, known as the Debye-Waller factor, is sensitive to statistical correlations among phonon coordinates via $\left\langle\hat{Q}_{\mathbf{k} s} \hat{Q}_{-\mathbf{k} s}\right\rangle$. For the case $s^{\prime}=s$ this is a measurement of the coordinate variance, provided $\left\langle\hat{Q}_{\mathbf{k} s}\right\rangle=$ $\left\langle\hat{Q}_{-\mathbf{k} s}\right\rangle=0$. Terms with $s^{\prime} \neq s$ correspond to covariances associated with "combination modes" [6].

Using x-ray diffraction to study the atomic position variance on a femtosecond time scale has been demonstrated for laser-induced destruction of organic films [8] and for ultrafast melting of semiconductors [9-13]. In these experiments, an intense optical "pump" pulse drives the crystal to a disordered state. Observation of squeezed phonons, however, requires the crystal to maintain its long range order after the pump pulse softens phonon frequencies on a time scale faster than the phonon period.

The Hamiltonian for a crystal lattice softened by a laser pulse arriving at a time $t=0$ in the harmonic approximation is

$$
\hat{H}(t)=\sum_{\mathbf{k}, s} \hbar \omega_{\mathbf{k} s}(t)\left(\hat{a}_{\mathbf{k} s}^{\dagger} \hat{a}_{\mathbf{k} s}+\frac{1}{2}\right),
$$

where $\omega_{\mathbf{k} s}(t)$ is the mode frequency and the operator $\hat{a}_{\mathbf{k} s}$ and its adjoint $\hat{a}_{\mathbf{k} s}^{\dagger}$ are the phonon annihilation and creation operators. We represent the evolution of the system in the Heisenberg picture as

$$
\hat{a}_{\mathbf{k} s}(t)=\sum_{s^{\prime}}\left[U_{\mathbf{k} s s^{\prime}}(t) \hat{a}_{\mathbf{k} s^{\prime}}\left(t_{\mathrm{eq}}\right)+V_{-\mathbf{k} s s^{\prime}}(t) \hat{a}_{-\mathbf{k} s^{\prime}}^{\dagger}\left(t_{\mathrm{eq}}\right)\right],
$$

where $t_{\text {eq }}<0$ is a particular time when the crystal is in thermal equilibrium and described by an admixture of phonon number states [14]. From this it is straightforward to show for an initial temperature $T$

$$
\begin{gathered}
\left\langle\left(\hat{\mathbf{u}}_{j} \cdot \mathbf{G}\right)^{2}\right\rangle=\sum_{\mathbf{k}, s^{\prime}} C_{\mathbf{k} s^{\prime}}\left|\sum_{s} \frac{\boldsymbol{\epsilon}_{\mathbf{k} s}^{j}(t) \cdot \mathbf{G}}{\sqrt{\omega_{\mathbf{k} s}(t)}}\left[U_{\mathbf{k} s s^{\prime}}(t)+V_{\mathbf{k} s s^{\prime}}(t)^{*}\right]\right|^{2} \\
C_{\mathbf{k} s^{\prime}}=\frac{\hbar}{2 N} \operatorname{coth} \frac{\hbar \omega_{\mathbf{k} s^{\prime}}\left(t_{\mathrm{eq}}\right)}{2 k_{B} T}
\end{gathered}
$$

for times $t>t_{\mathrm{eq}}$, where $k_{B}$ is the Boltzmann constant [15]. 
The tensors $U_{\mathbf{k} s s^{\prime}}(t)$ and $V_{\mathbf{k} s s^{\prime}}(t)$ can be computed for arbitrary $\omega_{\mathbf{k} s}(t)$ and $\boldsymbol{\epsilon}_{\mathbf{k} s}^{j}(t)$ using a recursion relation method to ensure continuity of the position and momentum operators [16]. For an instantaneous change $\omega_{\mathbf{k} s}^{(0)} \rightarrow$ $\alpha_{\mathbf{k} s} \omega_{\mathbf{k} s}^{(0)}=\omega_{\mathbf{k} s}^{(1)}$ and $\boldsymbol{\epsilon}_{\mathbf{k} s}^{j(0)} \rightarrow \boldsymbol{\epsilon}_{\mathbf{k} s}^{j(1)}$ at $t=0$ we find for $t>0$

$$
\begin{aligned}
\left\langle\left(\hat{\mathbf{u}}_{j} \cdot \mathbf{G}\right)^{2}\right\rangle= & \sum_{\mathbf{k}, s^{\prime}} C_{\mathbf{k} s^{\prime}} \mid \sum_{s} \frac{\boldsymbol{\epsilon}_{\mathbf{k} s}^{j(1)} \cdot \mathbf{G}}{\sqrt{\omega_{\mathbf{k} s}^{(1)}}} \sum_{j^{\prime}} M_{j^{\prime}}\left[\boldsymbol{\epsilon}_{\mathbf{k} s}^{j^{\prime}(1) *} \cdot \boldsymbol{\epsilon}^{\boldsymbol{H}^{\prime}}\right. \\
& \times\left.\frac{\omega_{\mathbf{k} s}^{(1)} \cos \omega_{\mathbf{k} s}^{(1)} t-i \omega_{\mathbf{k} s^{\prime}}^{(0)} \sin \omega_{\mathbf{k} s}^{(1)} t}{\sqrt{\omega_{\mathbf{k} s}^{(1)} \omega_{\mathbf{k} s^{\prime}}^{(0)}}}\right|^{2} .
\end{aligned}
$$

If $\boldsymbol{\epsilon}_{\mathbf{k} s}^{j(0)}=\boldsymbol{\epsilon}_{\mathbf{k} s}^{j(1)}$ this simplifies to

$$
\begin{aligned}
\left\langle\left(\hat{\mathbf{u}}_{j} \cdot \mathbf{G}\right)^{2}\right\rangle= & \frac{1}{2} \sum_{\mathbf{k}, s} C_{\mathbf{k} s} \frac{\left|\boldsymbol{\epsilon}_{\mathbf{k} s}^{j(0)} \cdot \mathbf{G}\right|^{2}}{\omega_{\mathbf{k} s}^{(0)}}\left[\left(\frac{1}{\alpha_{\mathbf{k} s}^{2}}+1\right)\right. \\
& \left.-\left(\frac{1}{\alpha_{\mathbf{k} s}^{2}}-1\right) \cos 2 \omega_{\mathbf{k} s}^{(1)} t\right] .
\end{aligned}
$$

The time dependence is characterized by a sum of oscillations at twice the frequency of phonons throughout the Brillouin zone (BZ), weighted in favor of low-frequency acoustic modes. These oscillations are dynamics of $\left\langle\hat{Q}_{\mathbf{k} s} \hat{Q}_{-\mathbf{k} s}\right\rangle$ arising from squeezed phonon states. If the eigenvectors are not constant, additional "combination mode" $\left\langle\hat{Q}_{\mathbf{k} s} \hat{Q}_{-\mathbf{k} s^{\prime}}\right\rangle$ terms with $s \neq s^{\prime}$ appear. In this case cosinelike oscillations at the sum and difference frequencies among the phonon branches contribute.

To investigate these dynamics with $\mathrm{x}$-ray diffraction we apply the asymmetric grazing incidence diffraction technique with femtosecond $\mathrm{x}$ rays from an electron beam slicing source [16-18]. The sample is a single crystal of bismuth repeatedly excited by a femtosecond laser pulse $(800 \mathrm{~nm}, 115 \mathrm{fs}, 1 \mathrm{kHz})$ with a fluence of $1.37 \pm$ $0.14 \mathrm{~mJ} / \mathrm{cm}^{2}$ and later probed at a controlled delay time with a short 140 fs pulse of $\mathrm{x}$ rays.

Figure 1 shows the rhombohedral unit cell of bismuth, along with relevant directions and their crystallographic designations. For this structure, Eq. (1) simplifies to

$$
I \propto \cos ^{2}\left(\mathbf{G} \cdot\left\langle\hat{\mathbf{r}}_{1}\right\rangle\right) e^{-\left\langle(\hat{\mathbf{u}} \cdot \mathbf{G})^{2}\right\rangle},
$$

where $\left\langle(\hat{\mathbf{u}} \cdot \mathbf{G})^{2}\right\rangle=\left\langle\left(\hat{\mathbf{u}}_{1} \cdot \mathbf{G}\right)^{2}\right\rangle=\left\langle\left(\hat{\mathbf{u}}_{2} \cdot \mathbf{G}\right)^{2}\right\rangle$. We have chosen the origin of $\left\langle\hat{\mathbf{r}}_{1}\right\rangle$ to be the point exactly between the two basis atoms, so that $\left\langle\hat{\mathbf{r}}_{1}\right\rangle=-\left\langle\hat{\mathbf{r}}_{2}\right\rangle$. In equilibrium at room temperature, $\left\langle\hat{\mathbf{r}}_{1}\right\rangle=z \mathbf{c}$, where $z=0.2334$ and $\mathbf{c}$ is a vector spanning the unit cell along the [111] direction [19]. Short-pulse laser excitation of bismuth causes the quasiequilibrium value of $z$ to change by a small amount, driving oscillations in $z(t)$ due to excitation of a $\mathbf{k}=0$ coherent $A_{1 g}$ phonon mode [18,20-23]. For diffraction from the (111) lattice planes, $\mathbf{G}=6 \pi \mathbf{c} /|\mathbf{c}|^{2}$. The diffracted intensity is then proportional to $\cos ^{2} 6 \pi z(t)$, resulting in modulation of the diffracted intensity at the $A_{1 g}$ frequency [see Fig. 2(a)].

For lattice planes perpendicular to the (111) planes, the coherent $A_{1 g}$ mode cannot contribute to the diffracted

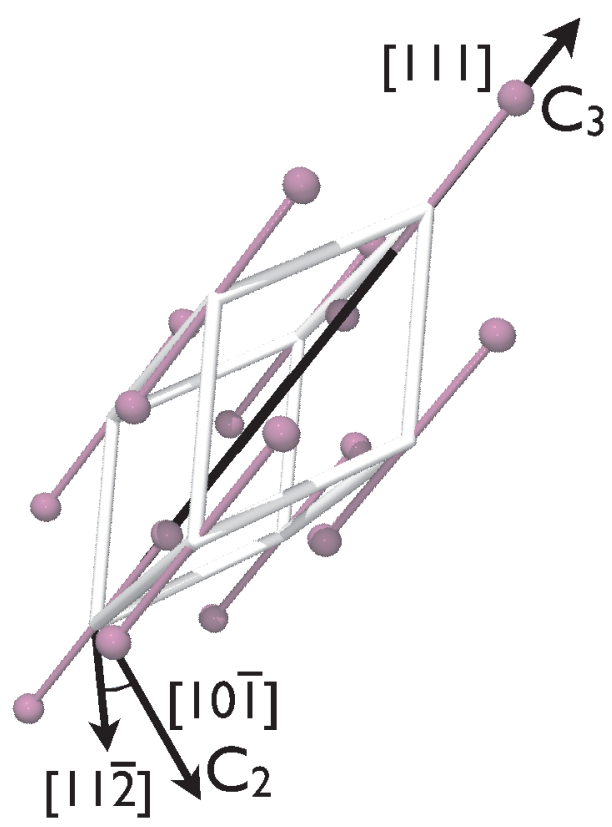

FIG. 1 (color online). Sketch of the bismuth unit cell, showing directions used for the diffraction measurements.

intensity because the reciprocal lattice vector $\mathbf{G}$ is perpendicular to the eigenvectors of the $A_{1 g}$ mode. Figure 2(b) shows data acquired for the (10) $\overline{1})$ and $(11 \overline{2})$ planes, each of which are perpendicular to (111). Both sets of room temperature data show similar behavior: a decrease in intensity and a highly damped cosinelike oscillation with a period of approximately $750 \mathrm{fs}$. The magnitude of the change scales approximately with the square of the magnitude of the reciprocal lattice vector: $\left|\mathbf{G}_{11 \overline{2}}\right|^{2} /\left|\mathbf{G}_{10 \overline{1}}\right|^{2}=3.00$. On cooling the crystal to $170 \mathrm{~K}$, the magnitude of the drop in the (11) $)$ peak decreases by about a factor of 2 .

Although it is in principle possible for coherent $E_{g}$ optical phonon modes to contribute to diffraction from these lattice planes, we may discount this as a source of the observed oscillations. Coherent $E_{g}$ modes correspond to changes in $\left\langle\hat{\mathbf{r}}_{1}\right\rangle$ in the plane perpendicular to [111]. The relevant phonon coordinate is $x(t)=\mathbf{G} \cdot\left\langle\mathbf{r}_{1}(t)\right\rangle$. From Eq. (8), the diffracted intensity is proportional to $\cos ^{2} x(t)$. Impulsive Raman excitation of an $E_{g}$ mode leads to oscillation of $x(t)$ about 0 with a frequency of approximately $1.6 \mathrm{THz}$ at our excitation levels [21]. This would cause oscillation in the diffracted $\mathrm{x}$-ray intensity with a frequency of $3.2 \mathrm{THz}$, much faster than the $1.3 \mathrm{THz}$ observed. The fast damping time of the observed oscillations is also inconsistent with previous observations of coherent $E_{g}$ phonons observed optically under similar excitation conditions $[21,24]$. We conclude that coherent $E_{g}$ phonons are too small in magnitude to contribute significantly to our data.

The remaining possible contribution is from the Debye-Waller factor. Figure 2(c) shows the behavior of the change in the direction-projected mean-square dis- 


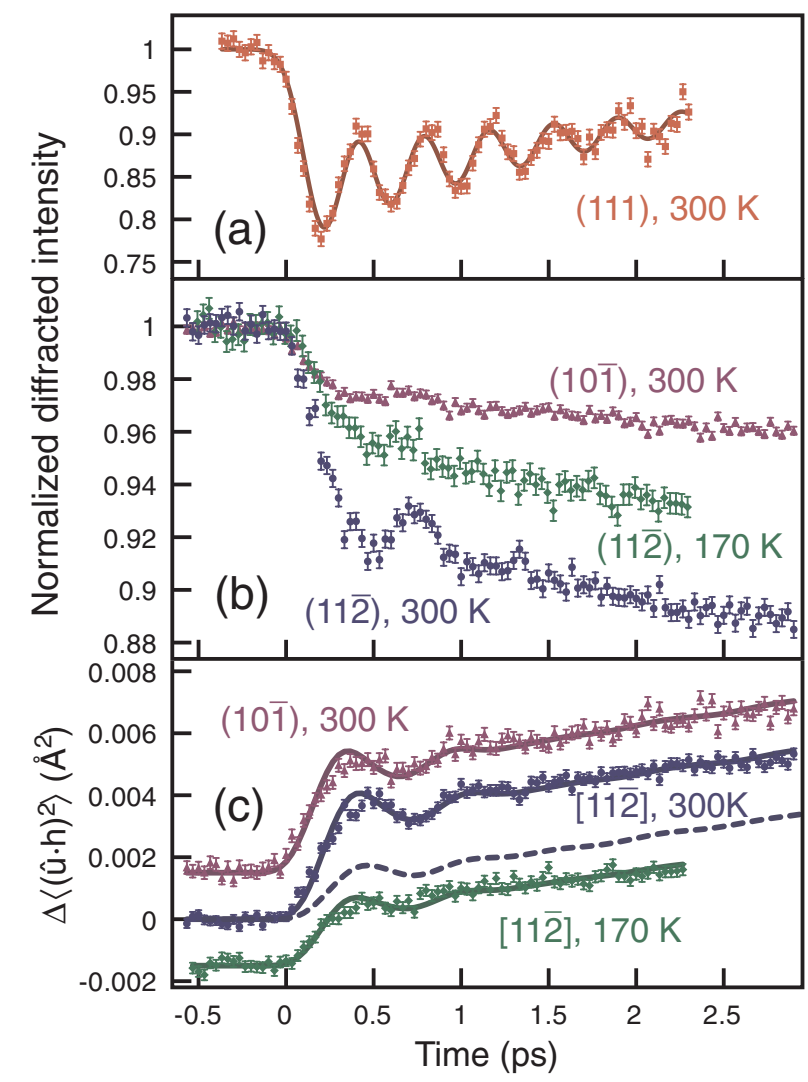

FIG. 2 (color online). (a) Time-resolved diffraction from the (111) lattice planes, showing the coherent $A_{1 g}$ mode. The solid line shows a fit using a constant frequency model [18] which is also used to estimate the pump pulse arrival time $t=0$. (b) Diffraction signal from the (101) planes at room temperature (purple triangles) and the $(11 \overline{2})$ planes at both room temperature (blue circles) and at $170 \mathrm{~K}$ (green diamonds). (c) Change in the direction-projected mean-square atomic displacement for [10ī] at room temperature (purple triangles), and [112] at room temperature (blue circles) and at $170 \mathrm{~K}$ (green diamonds). For

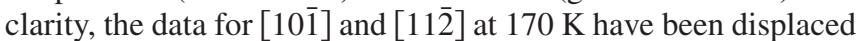
vertically. The solid lines are the results of the fit discussed in the text. The dashed blue line is the prediction for the [112] direction at room temperature based on DFT [26].

placement $\left\langle(\hat{\mathbf{u}} \cdot \mathbf{h})^{2}\right\rangle$ inferred from the data, where $\mathbf{h}=$ $\mathbf{G} /|\mathbf{G}|$. Both sets of room temperature data show nearly identical behavior: a rise of about $0.003 \AA^{2}$ accompanied by a highly damped oscillation, followed by a slower, nearly linear increase. At room temperature, the equilibrium value of $\left\langle(\hat{\mathbf{u}} \cdot \mathbf{h})^{2}\right\rangle$ is $0.0143 \AA^{2}$ [25]. The smaller change in $\left\langle(\hat{\mathbf{u}} \cdot \mathbf{h})^{2}\right\rangle$ observed in the [11 $\left.\overline{2}\right]$ direction on cooling the sample is consistent with the $T$ dependence of Eqs. (4) and (5).

To model the data, we evaluate Eq. (4) using the ground and excited state phonon frequencies and eigenvectors calculated from first-principles density functional theory (DFT) [26]. These calculations employ a model of carrier excitation in which $1.0 \%$ of the valence band electrons per cell are promoted to the conduction band. The carriers are placed into a statistical distribution with separate chemical potentials for the holes and electrons, and the electron-hole density is a constant of motion. This is in contrast to another DFT model for excited bismuth by Zijlstra et al. which calculated the dynamics of the $\Gamma$-point optical phonon coordinates assuming the carriers share a common chemical potential and the electronic entropy is a constant of motion [27]. The "two chemical potential" DFT model we have used predicts that this level of electronic excitation causes an approximately $10 \%$ decrease in the frequency of all the optical modes and of the acoustic phonon modes near the $X$ point at the edge of the BZ, but relatively little change in the frequency of acoustic modes in other regions in the BZ. The DFT excited state $A_{1 g}$ phonon frequency of $2.61 \mathrm{THz}$ matches the frequency of $2.61 \pm 0.01 \mathrm{THz}$ estimated by a fit to the (111) diffraction data assuming a constant $A_{1 g}$ frequency [see Fig. 2(a)] [18].

For our calculation of $\left\langle(\hat{\mathbf{u}} \cdot \mathbf{h})^{2}\right\rangle$, we model the frequency softening and eigenvector change as an instantaneous change at a time $t_{p}$ [28]. The predicted initial equilibrium value of $\left\langle(\hat{\mathbf{u}} \cdot \mathbf{h})^{2}\right\rangle$ is $0.0214 \AA^{2}$, somewhat higher than the experimental value of $0.0143 \AA^{2}$ [25]. Incoherent coupling of electronic energy to the lattice is modeled as an additive linear contribution $b\left(t-t_{p}\right)$ that begins at $t_{p}$. The result is then convolved with a Gaussian with full width at half maximum equal to the estimated time resolution of $200 \mathrm{fs}$. The dashed line in Fig. 2(c) shows the calculated change in the mean-square displacement for [112̄] with $T=300 \mathrm{~K}, t_{p}=0.1 \mathrm{ps}$ and $b=$ $8 \mathrm{pm}^{2} / \mathrm{ps}$. The corresponding curve for [101] at $300 \mathrm{~K}$ is identical. The oscillation frequency and dephasing time match the data within the uncertainties. The calculated curve is the sum of many cosinelike oscillations over a continuum of frequencies corresponding to phonon modes that have been softened by the pump excitation. The oscillation frequency is twice the average of these softened mode frequencies, weighted according to the frequencyand eigenvector-dependent prefactors in Eq. (4) that magnify the contribution of low-frequency modes. The dephasing rate is determined by the width of the frequency distribution of these modes, due primarily to $\mathbf{k}$-space dispersion of the acoustic bands. The rate of dephasing in the measured data may also be affected by depth inhomogeneities [18], but this can be estimated as a much smaller contribution: approximately $10 \%$ of the oscillation frequency for a $10 \%$ mode softening. Despite agreement with the data on the shape of the observed transient, the model underestimates the magnitude of the change by a factor of approximately 2.5 . In this calculation we have not taken into account possible dynamical effects due to the large amplitude coherent $A_{1 g}$ motion which may modulate the frequencies of other phonon modes. Also, anharmonic coupling of the $A_{1 g}$ mode to acoustic modes can also play a role. While these effects might help to explain the discrepancy, the lack of a $2.61 \mathrm{THz}$ frequency component in the

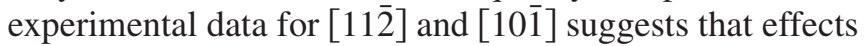


TABLE I. Fit parameters for the constant-fraction softening model discussed in the text, for the three data sets shown in Fig. 2(c). The small change of the fitted value of $t_{p}$ for different directions is within the estimated range of experimental timing precision $( \pm 50 \mathrm{fs}$ ) caused by small changes in the pump-probe overlap point from the rotation of the sample to access different diffraction peaks.

\begin{tabular}{ccccc}
\hline \hline Direction & $T(K)$ & $\alpha$ & $b\left(\mathrm{pm}^{2} / \mathrm{ps}\right)$ & $t_{p}(\mathrm{ps})$ \\
\hline$[10 \overline{1}]$ & 300 & $0.884 \pm 0.002$ & $8.8 \pm 0.3$ & $0.054 \pm 0.006$ \\
{$[11 \overline{2}]$} & 300 & $0.8793 \pm 0.0013$ & $8.0 \pm 0.2$ & $0.117 \pm 0.004$ \\
{$[11 \overline{2}]$} & 170 & $0.889 \pm 0.003$ & $7.4 \pm 0.4$ & $0.105 \pm 0.009$ \\
\hline \hline
\end{tabular}

from interaction with the $A_{1 g}$ mode are smaller than the experimental uncertainties. Double-pump excitation experiments, where the population of the coherent $A_{1 g}$ mode is varied but the electronic excitation is kept constant, also show no significant influence of the $A_{1 g}$ population on the magnitude of the drop in (112) diffraction at room temperature for times $>1$ ps after excitation [29].

As an alternative to the DFT results, we have computed the time dependence of $\left\langle(\hat{\mathbf{u}} \cdot \mathbf{h})^{2}\right\rangle$ for a simple semiempirical model where the phonon frequencies change at time $t_{p}$ by a fixed fractional amount regardless of their position in $\mathbf{k}$-space: $\omega_{\mathbf{k} s} \rightarrow \alpha \omega_{\mathbf{k} s}$. The eigenvectors are kept fixed to their initial values. This model is motivated intuitively by the idea that moving electrons from the valence bands to the conduction bands weakens interatomic bonds, and as a first approximation we assume this impacts all phonon modes equally. The solid lines in Fig. 2(c) show a fit of this model to the data, achieved by varying $\alpha$, the slope $b$ of the linear incoherent electron-phonon contribution, and the pump arrival time $t_{p}$. Table I summarizes the results. The main features of the data are well described by this model, with similar values of the fit parameters. As with the DFT-based model, the frequency and dephasing time are determined by the excited acoustic phonon dispersion. Interestingly, the fitted values of $\alpha \approx 0.88$ are very close to the observed softening of the coherent $A_{1 g}$ mode, where the frequency decreases to 0.89 of its initial value at room temperature. The model places the initial maximum in $\left\langle(\hat{\mathbf{u}} \cdot \mathbf{h})^{2}\right\rangle$ at approximately 150 fs earlier than the measurements indicate. This may indicate a delay in the softening of the phonon modes from an approximately $260 \mathrm{fs}$ electron-hole interaction time estimated from earlier measurements [18].

The data shown here demonstrate phonon squeezing by using femtosecond $\mathrm{x}$-ray diffraction to probe directly the direction-projected mean-square atomic position variance. At lower sample temperatures $(<30 \mathrm{~K})$ it has potential to demonstrate for the first time vacuum state squeezing of the phonon field, where the variance is suppressed below the zero-point motion [4]. For the present experiment, agreement between a simple semiempirical model and the data suggests that femtosecond laser excitation of bismuth causes significant softening of the acoustic modes throughout the BZ, a result that appears to contradict the predictions of a model based on first-principles DFT.

These experiments were performed on the X05LA beam line at the Swiss Light Source, Paul Scherrer Institut, Villigen, Switzerland. We thank D. Grolimund and C. Borca for assistance, and we acknowledge R. Merlin, D. Reis, and E. S. Zijlstra for fruitful discussions.

*steve.johnson@psi.ch

[1] V. Dodonov, J. Opt. B 4, R1 (2002).

[2] D. Walls, Nature (London) 306, 141 (1983).

[3] G. A. Garrett et al., Science 275, 1638 (1997).

[4] X. D. Hu and F. Nori, Physica (Amsterdam) 263B, 16 (1999).

[5] O. V. Misochko, K. Sakai, and S. Nakashima, Phys. Rev. B 61, 11225 (2000).

[6] A. Bartels, T. Dekorsy, and H. Kurz, Phys. Rev. Lett. 84, 2981 (2000).

[7] Equation (1) assumes translational symmetry, preserved for spatially homogeneous excitation.

[8] C. Rischel et al., Nature (London) 390, 490 (1997).

[9] A. Rousse et al., Nature (London) 410, 65 (2001).

[10] K. Sokolowski-Tinten et al., Phys. Rev. Lett. 87, 225701 (2001).

[11] A. M. Lindenberg et al., Science 308, 392 (2005).

[12] K. Gaffney et al., Phys. Rev. Lett. 95, 125701 (2005).

[13] P. B. Hillyard et al., Phys. Rev. Lett. 98, 125501 (2007).

[14] T. Kiss, J. Janszky, and P. Adam, Phys. Rev. A 49, 4935 (1994).

[15] We also use the relations $\omega_{\mathbf{k} s}=\omega_{-\mathbf{k} s}$ and $\boldsymbol{\epsilon}_{\mathbf{k} s}^{j}=\left(\boldsymbol{\epsilon}_{-\mathbf{k} s}^{j}\right)^{*}$.

[16] See EPAPS Document No. E-PRLTAO-102-054914 for additional information on the technical details of the femtosecond x-ray diffraction experiment and a mathematical derivation of the recursion relations used to simulate the data. For more information on EPAPS, see http:// www.aip.org/pubservs/epaps.html.

[17] P. Beaud et al., Phys. Rev. Lett. 99, 174801 (2007).

[18] S. L. Johnson et al., Phys. Rev. Lett. 100, 155501 (2008).

[19] P. Fischer, I. Sosnowska, and M. Szymanski, J. Phys. C 11, 1043 (1978).

[20] H. Zeiger et al., Phys. Rev. B 45, 768 (1992).

[21] M. Hase et al., Phys. Rev. Lett. 88, 067401 (2002).

[22] K. Sokolowski-Tinten et al., Nature (London) 422, 287 (2003).

[23] D. Fritz et al., Science 315, 633 (2007).

[24] O. V. Misochko et al., J. Phys. Condens. Matter 18, 10571 (2006).

[25] M. S. Narayana and N. G. Krishna, Phys. Status Solidi A 202, 2731 (2005).

[26] É. D. Murray et al., Phys. Rev. B 75, 184301 (2007).

[27] E. S. Zijlstra, L. L. Tatarinova, and M.E. Garcia, Phys. Rev. B 74, 220301(R) (2006).

[28] Keeping eigenvectors fixed at their initial values showed no differences within experimental uncertainties, implying combination modes do not contribute significantly.

[29] P. Beaud et al., in Ultrafast Phenomena XVI, edited by P. Corkum et al. (Springer, New York, 2009), p. 104. 